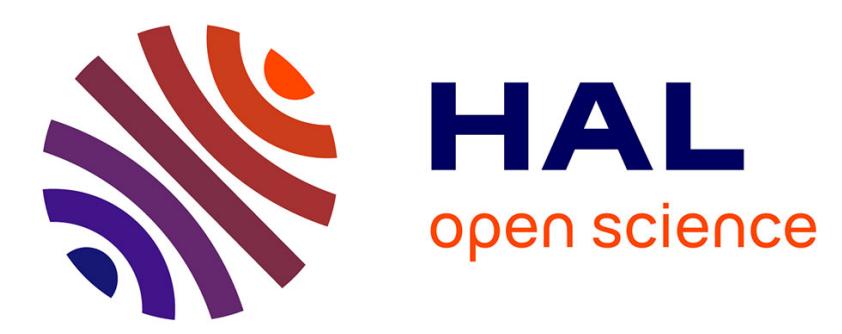

\title{
Anisotropy and damping of molecules/cobalt hybrid thin films
}

\author{
Olivier Rousseau, Salim Cherif, Yves Roussigne, Mohamed Belmeguenai, \\ Pascal Martin, Jean-Christophe Lacroix, Maria Luisa Della Rocca, Philippe \\ Lafarge, Clement Barraud
}

\section{To cite this version:}

Olivier Rousseau, Salim Cherif, Yves Roussigne, Mohamed Belmeguenai, Pascal Martin, et al.. Anisotropy and damping of molecules/cobalt hybrid thin films. IEEE Transactions on Magnetics, 2017, pp.1 - 1. 10.1109/TMAG.2017.2694888 . hal-01549026

\section{HAL Id: hal-01549026 \\ https://hal-univ-paris.archives-ouvertes.fr/hal-01549026}

Submitted on 28 Jun 2017

HAL is a multi-disciplinary open access archive for the deposit and dissemination of scientific research documents, whether they are published or not. The documents may come from teaching and research institutions in France or abroad, or from public or private research centers.
L'archive ouverte pluridisciplinaire HAL, est destinée au dépôt et à la diffusion de documents scientifiques de niveau recherche, publiés ou non, émanant des établissements d'enseignement et de recherche français ou étrangers, des laboratoires publics ou privés. 


\title{
Anisotropy and damping of molecules/cobalt hybrid thin films
}

\author{
O. Rousseau ${ }^{1}$, S. M. Chérif ${ }^{1 *}$, Y. Roussigné ${ }^{1}$, M. Belmeguenai ${ }^{1}$, P. Martin ${ }^{2}$, J-C. Lacroix ${ }^{2}$, M. L. Della

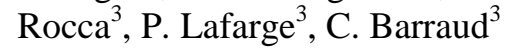

\begin{abstract}
We have investigated the magnetic properties of evaporated Co thin films covalently functionalized with different organic thin films, namely (1-(2-bisthienyl benzene) and nitro-benzene. The coating is realized thanks to a diazonium-based electro-reduction process. Brillouin light scattering experiments revealed that the magnetic properties are sensitive to the presence of the organic film. For (1-(2-bisthienyl benzene) thin films, the perpendicular magnetic anisotropy is increased as well as the magnetic damping. However for nitrobenzene, only the perpendicular anisotropy is increased albeit less than for (1-(2-bisthienyl benzene). This change in magnetic properties might be attributed to the coupling with the organic molecules.
\end{abstract}

Index Terms - Anisotropy, Damping, Organic molecules, Cobalt thin films, Brillouin light scattering.

\section{INTRODUCTION}

$I^{2}$ $n$ the last decades, there is a considerable effort in research to control the properties of magnetic materials and spins of electrons for spintronics applications as memories [1-7]. The classical method is to combine magnetic thin layers with different properties or to tune an alloy composition $[3,4,8,9]$. Another emerging possibility is the coupling of a ferromagnet with organic molecules. Organic molecules can affect the surface magnetic properties mainly through interfacial effects $[2,5,6,10-13]$. Currently, the achievable thickness of the ferromagnetic layers is thin enough, in $\mathrm{nm}$ range, to achieve enhancement of the magnetic properties through the interfacial interaction. The interest of organic layers is the variety of means to reliably add them in devices. The most used deposition techniques are the evaporation [1], the selfassembly of molecular monolayers [2] and the electrochemical grafting (electrochemical coating) [14]. In case of

ANR (Agence Nationale de la Recherche) and CGI (Commissariat à l'Investissement d'Avenir) are gratefully acknowledged for their financial support of this work through Labex SEAM (Science and Engineering for Advanced Materials and devices), HEFOR project (PRS-G-2014).”

O. Rousseau, Y. Roussigné., M. Belmeguenai and S.M. Chérif are with Laboratoire des Sciences des Procédés et des Matériaux (UPR 3407), Université Paris 13-Nord, Sorbonne Paris Cité, CNRS, 93430, Villetaneuse, France (e-mail : cherif@univ-paris13.fr).

P. Martin and J-C. Lacroix are with Laboratoire Interfaces Traitements Organisation et DYnamique des Systèmes (UMR 7086), Université Paris Diderot, Sorbonne Paris Cité, CNRS, 75205 Paris Cedex 13, France (e-mail : (pascal.martin@univ-paris-diderot.fr).

M. L. Della Rocca, P. Lafarge and C. Barraud are with Laboratoire Matériaux et Phénomènes Quantiques (UMR 7162), Université Paris Diderot, Sorbonne Paris Cité, CNRS, 75205 Paris Cedex 13, France (e-mail: clement.barraud@univ-paris-diderot.fr). electrochemical coating, the organic molecule is covalently bonded to the ferromagnetic layer's surface. Stronger coupling [15] and thus tuning of the properties is expected compared to the physisorption case obtained by evaporation. Ab-initio calculations $[10,16,17]$ demonstrate that what is important is the delocalized electrons as the conduction band of graphene [18]. In molecules, this corresponds to the $\pi$-bands which tend to strongly hybridize at the surface with a metal. Adsorption of molecules on a ferromagnetic surface can also induce molecular roughening causing substantial changes in anisotropies and spin polarization [19]. However, the experimental characterization of magnetization dynamics when coupled to organic molecules has to be done for development of organic spintronic devices. A well-known non-invasive tool for probing magnetization dynamics is the Brillouin light scattering (BLS) [20-22].

In this paper, we report measurements of BLS spectra of a Co layer coated by either 1-(2-bisthienyl) benzene (BTB) or nitro benzene (NB) through the same diazonium-based electrochemistry process. We found that the magnetic properties are strongly tuned when the molecule is rich in $\pi$ electrons. Our results demonstrate that the magnetic anisotropy as well as the damping can be tuned by the appropriated organic molecule.

\section{EXPERIMENTAL}

Chemicals: 1-(2-bisthienyl)-4-aminobenzene (BTB) was synthesized according to published procedures [23]. All chemicals were purchased from Sigma Aldrich and used as received. Lithium perchlorate $\left(\mathrm{LiClO}_{4}\right)$ and tetrabutylammonium tetrafluoroborate $\left(\mathrm{Bu}_{4} \mathrm{NBF}_{4}\right)$ were used as supporting electrolyte at $0.1 \mathrm{M}$ concentration in acetonitrile (ACN).

Ti $(1 \mathrm{~nm}) / \mathrm{Pt}(20 \mathrm{~nm}) / \mathrm{Co}(8 \mathrm{~nm})$ heterostructures were deposited by electron-beam evaporation under high vacuum conditions $\left(10^{-8} \mathrm{mBar}\right)$ on $\mathrm{Si} / \mathrm{SiO}_{2}$ substrate. Low deposition rates $(0.05 \mathrm{~nm} / \mathrm{s})$ were chosen.

For the electrochemical experiments a conventional threeelectrode cell was used. Platinum wire was used as auxiliary electrode, saturated calomel electrode (SCE) was used as reference electrode. Before any electrochemical measurements the solutions were deoxygenated by bubbling argon gas for 30 minutes, during the experiment the electrochemical cell remain under argon. The potentiostat used in this study was a CHI 660C (CH Instruments, made in USA).

Electrochemical deposition of the organic layer: Before electrochemical coating, the cobalt electrodes were immersed in an acidic solution and a negative potential was applied 
during 10 seconds to remove the $\mathrm{CoO}$ oxide layer. The cobalt layer was then modified by either 1-(2-bisthienyl) benzene (BTB) or nitro benzene (NB) through the same diazoniumbased electrochemistry process. In the case of $\mathrm{NB}$, a solution of $0.5 \mathrm{mM}$ of nitrobenzene diazonium salt (NBD) in ACN with $0.1 \mathrm{M} \mathrm{Bu}_{4} \mathrm{NBF}_{4}$ was prepared for the electrochemical coating $[24,25]$. In the case of BTB, on the contrary, the formation of the diazonium cation from BTB precursor was performed in situ [26]. Briefly, an ACN solution containing of 1-(2-bisthienyl)-4-aminobenzene precursor $(0.5 \mathrm{mM})$ and $\mathrm{Bu}_{4} \mathrm{NBF}_{4}(0.1 \mathrm{M})$ as supporting electrolyte was prepared and degassed by argon bubbling for at least 10 minutes. Following that, $\mathrm{tBuO}-\mathrm{NO}$ (30 equivalents) was added in excess to the solution. As the reaction between amine function and tertbutyl nitrite is relatively slow, coating was only started after 5 minutes incubation time. NB and BTB layer deposition was performed on glassy carbon electrodes as well as on cobalt electrodes using cycling voltammetry (Fig. 1). The number of cycles gives us a thickness of around $7 \pm 0.6 \mathrm{~nm}$ for both depositions.

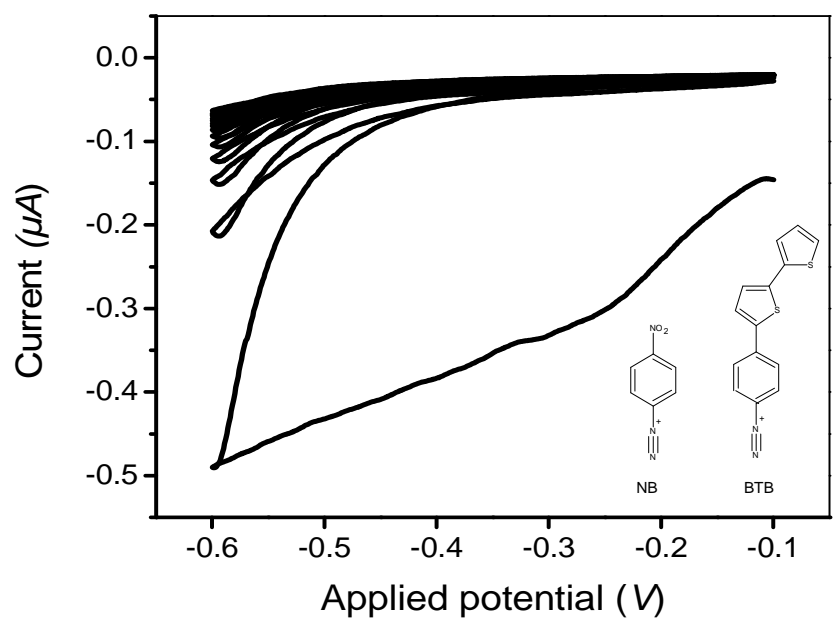

Fig.1: Cyclic voltammetry on cobalt substrate for the reduction of NB diazonium salt $\left(5 \times 10^{-4} \mathrm{~mol} \mathrm{~L}^{-1}\right)$ in ACN with $0.1 \mathrm{M}$ of supporting electrolyte. 10 cycles at scan rate 0.1 V.s. ${ }^{-1}$, inset: structures of NB and BTB diazonium salts.

After the coating of the molecules, we measured the magnetization dynamics behavior of the samples using a conventional BLS technique.

The BLS technique gives access to magnon modes with nonzero wavevector values. In the BLS experiment, the magnons of a wavenumber $k$ in the range $0-20 \mu^{-1}$ (depending on the incidence angle $\theta$ : in backscattering configuration), are probed by illuminating the sample with a laser having a wavelength $\lambda=532 \mathrm{~nm}$. The laser power has been adapted in order to avoid any irremediable degradation of the molecules. The magnetic field was applied perpendicular to the incidence plane, which allows for probing excitons propagating along the in-plane direction perpendicular to the applied field: Damon-Eshbach (DE) geometry. The Stokes (S, negative frequency shift relative to the incident light as a magnon was created) and anti-Stokes (AS, positive frequency shift relative to the incident light as a magnon was absorbed) frequencies, are detected simultaneously. BLS spectra of ferromagnetic films can be characterized by a pronounced Stokes/Anti-Stokes intensity asymmetry that is known to be a peculiarity of time-reversal symmetry and that is reversed under the reversed bias. The probed magnons were studied as a function of applied magnetic field (up to $200 \mathrm{mT}$ ). Frequencies and the peaks linewidth (full width at half maximum: $F W H M$ ) were determined from Lorentzian fits to the BLS spectra.

\section{RESUlTS AND DisCUSSIONS}

\section{A. Nitrobenzene (NB) measurements}

We measured BLS spectra of the non-coated and NB coated areas of the Co film, with an incident angle of $35^{\circ}$, corresponding to a wavenumber $k$ of $13.5 \mu \mathrm{m}^{-1}$. Figure 2 shows typical obtained raw spectra (Stokes side). The magnons peaks were visible in both areas of the sample. In the Co area, the magnons peak is at higher frequency than in the coated area. It is to mention that we hardly observe magnons peak in the large area where the coating is fully achieved. This point is under investigations.

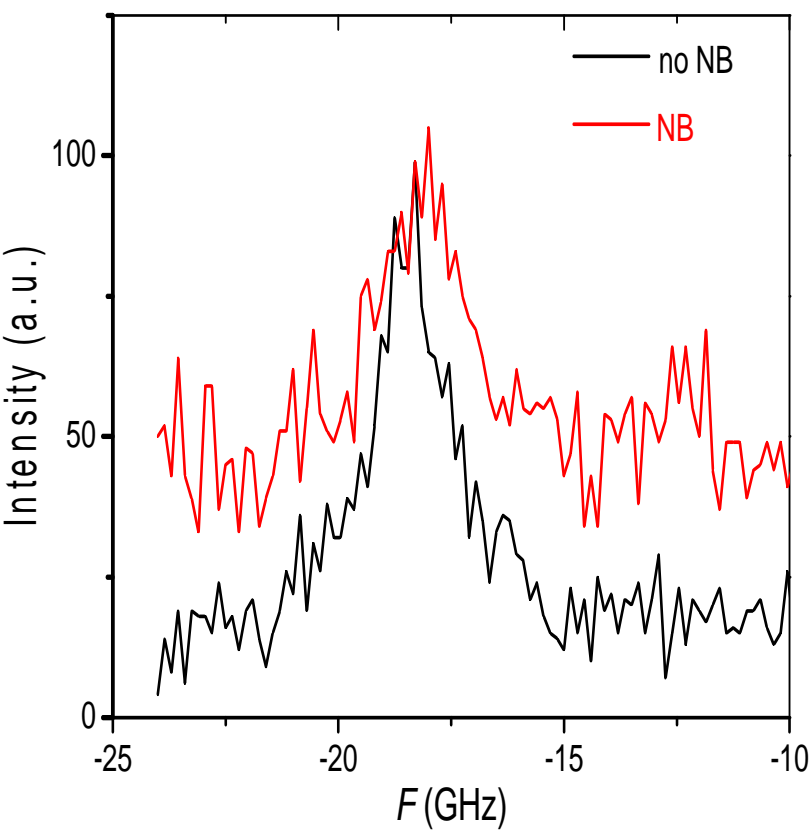

Fig. 2 (Colour online) Raw BLS spectra (Stokes side) obtained under a 200 $\mathrm{mT}$ in plane magnetic field and a wavenumber $k$ equal to $13.5 \mu \mathrm{m}^{-1}$; black: $\mathrm{Co}$ area (with presence of $\mathrm{CoO}$ ); red: area coated with NB molecules.

Figure 3 exhibits the experimental magnons frequency (fig. $3 a)$ and the peaks linewidth $F W H M$ (fig. 3b) evolution as function of the external magnetic field. As expected, the measured frequencies on the two areas are clearly shifted for all used values of magnetic field.

The magnons frequency is given by the dispersion law [27]: $F=\mu_{0} \frac{\gamma}{2 \pi} \sqrt{\left(H+J k^{2}+P(k t) M_{s}\right)\left(H+J k^{2}-P(k t) M_{s}+M_{s}-H_{K}\right)}$ where $H$ is the in-plane applied field, $M_{\mathrm{s}}$ is the saturation magnetization, $t$ is the ferromagnetic layer thickness, $\gamma$ is the gyromagnetic coefficient $\chi(2 \pi)=30 \mathrm{GHz} / \mathrm{T}, \mu_{0}$ is the permeability of vacuum, $J=2 A_{\mathrm{ex}} / \mu_{0} M_{\mathrm{s}}$, with $A_{\mathrm{ex}}$ the exchange stiffness constant of the magnetic material, $H_{\mathrm{K}}$ is the 
perpendicular uniaxial anisotropy field $\left(H_{\mathrm{K}}=2 K / M_{\mathrm{s}}\right.$, where $K$ is the anisotropy constant), and $P(k t)=1-\frac{1-\exp (-k t)}{k t}$. The coefficient $P(k t)$, describing dipolar interactions, reduces in thin films $(k t<<1)$ to a simple $k t / 2$, which makes this term linear in $k t$. It should be noticed that $k t \approx 0.11$ in our case. It is to notice that the frequency of the probed Damon-Eshbach (DE) surface mode given by (1) is largely insensitive to whether the exchange constant $A_{\text {ex }}$ is assumed or fitted.

A change of frequency, at fixed $k$ wavenumber and magnetic field $H$, should correspond to either a change in the magnetic anisotropy or a change in the Co thickness. The question is to know which one is dominant as potential factor in this change. This is discussed later in this paper. Concerning the magnons damping, the peaks linewidth $F W H M$, represented in fig. $3 \mathrm{~b}$, did not show change, within the considered experimental uncertainties, between the two investigated areas.
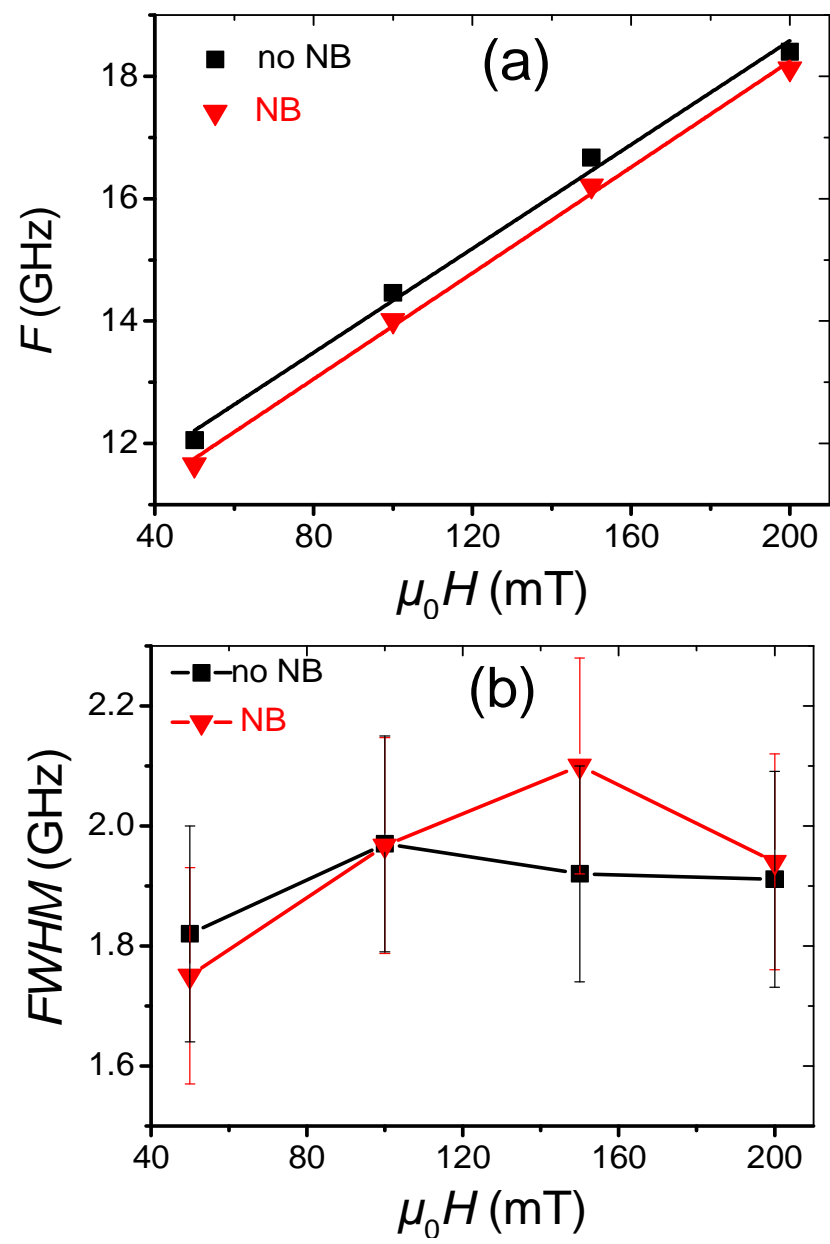

Fig. 3 (Colour online) Evolution of the magnons frequency (a) and of the linewidth (b) versus applied magnetic field. The data points acquired over the Co area are in black and over the area with NB molecules coated in red. The continuous lines are guide to eyes.

\section{B. 1-(2-bisthienyl) benzene (BTB) measurements}

We present now the results obtained with BTB molecules. In a second step we will compare them to those obtained with NB molecules, for the same experimental conditions.
Figure 4 shows typical raw BLS spectra (anti-Stokes side) for BTB coated and non-coated areas of the Co film. As for the NB molecules, the magnons are measured over both areas. One can observe that for this latter area the frequencies are lower than those measured in the Co area without BTB molecules coating. On the other hand, the experimental linewidth $(F W H M)$ is measured about $2.2 \mathrm{GHz}$ in the area coated with molecules while it is only about $1.2 \mathrm{GHz}$ in the non-coated one, for all the investigated range of the applied magnetic field.

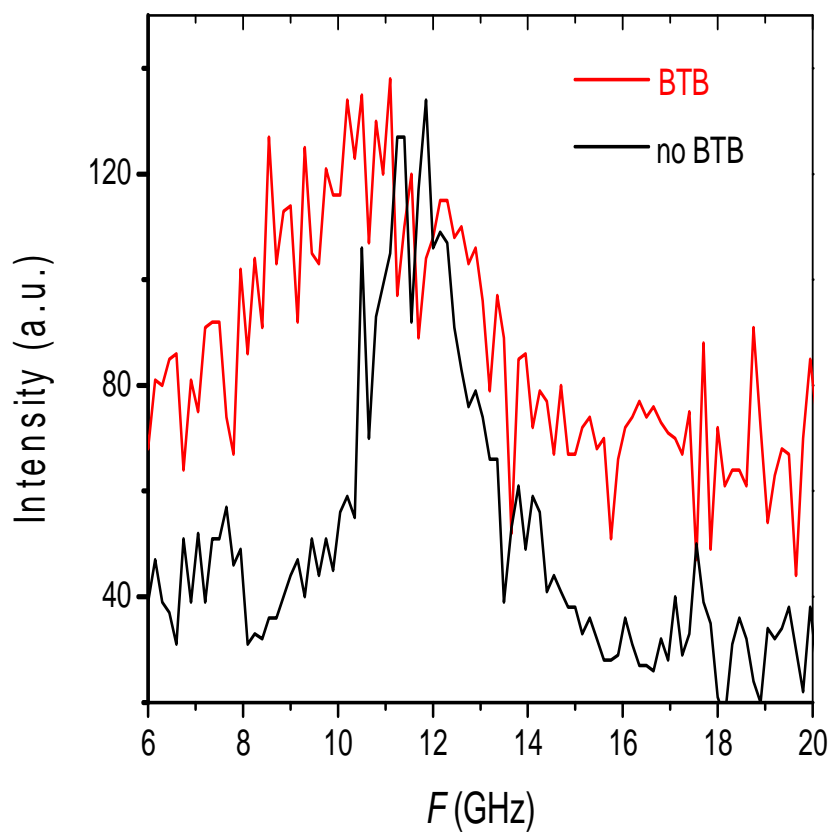

Fig. 4 (Colour online) Raw BLS spectra (anti-Stokes side) obtained under a $100 \mathrm{mT}$ in plane magnetic field and a wavenumber $k$ equal to $13.5 \mu^{-1}$; black: $\mathrm{Co}$ area (with presence of $\mathrm{CoO}$ ); red: area coated with BTB molecules.

Figure 5 shows the evolution of the measured frequencies as function of the applied magnetic field for an angle of incidence of $35^{\circ}$ corresponding to a wavenumber $k=13.5$ $\mu \mathrm{m}^{-1}$. We can observe that in this case the frequency shift between the two investigated areas is greater than for the NB sample, indicating a stronger effect of the coating of BTB molecules comparing to the NB molecules.

We now discuss the change of frequency in NB and BTB modified areas. First, the frequency of the pure Co magnons is different even between the two samples. At $200 \mathrm{mT}$, the frequency is about $18 \mathrm{GHz}$ for the NB sample while it is only about $15 \mathrm{GHz}$ for the BTB sample. The Co thin films came from different fabrication batches and thus their degree of oxidation might be different. Therefore, the two investigated Co samples present different properties. It is to note that the oxidation of the Co from oxygen of air reaches a passive state without any further evolution. Indeed, we checked that magnons peaks have the same frequency for the same sample and experimental conditions, even after several months at air. If we consider that the change of the frequencies in the presence of molecules over the covered area is solely because of change in the Co thickness, we obtained a huge difference 
in the thickness, frequency, after coating, between NB and BTB. However as the electrochemical coating proceeds from the same diazonium electrochemistry the same change in thickness and thus frequency is expected.

As we did not observe the same frequency difference $\delta F$ between coated and non-coated areas for BTB $(\delta F \approx 1.3 \mathrm{GHz})$ and $\mathrm{NB}(\delta F \approx 0.5 \mathrm{GHz})$ molecules, this frequency shift can be at least partially attributed to an effective anisotropy variation. In fact, the coated samples present the same nominal thickness then the probable way to modify the effective anisotropy is a variation of the surface anisotropy. In order to analyze the observed behavior, we roughly estimated the variation of the effective anisotropy by using equation (1).

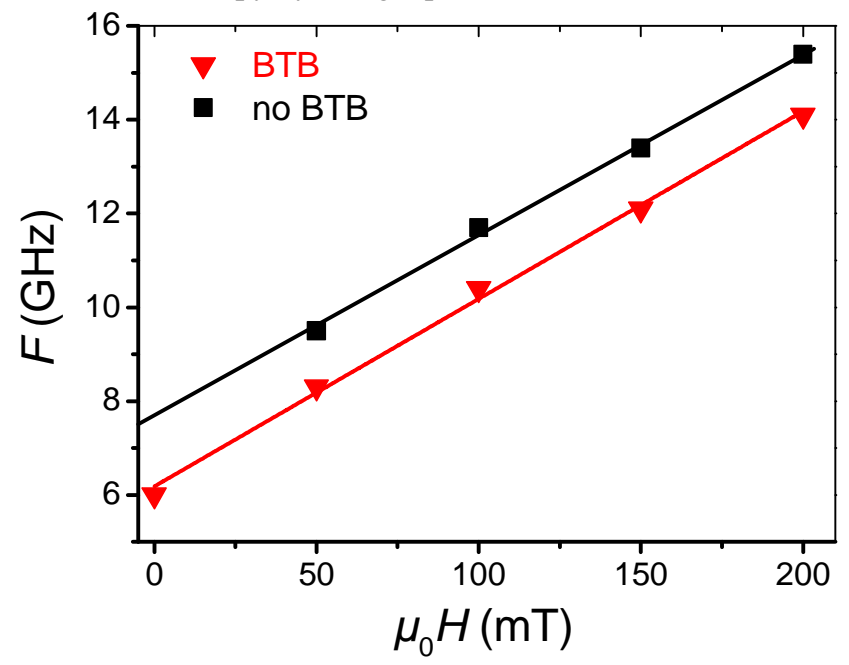

Fig. 5 (Colour online) Evolution of the magnons frequency versus applied magnetic field. The data points acquired over the Co area are in black and over the area coated with BTB molecules in red. The continuous lines are guide to eyes.

Let us first consider the case of BTB coated sample. We hypothesize that cobalt has the properties of bulk material with the following parameters: $\mathcal{H}(2 \pi)=30 \mathrm{GHz} / \mathrm{T} ; A_{\mathrm{ex}}=2 \times 10^{-11}$ $\mathrm{J} / \mathrm{m}$ and $\mu_{0} M_{\mathrm{s}}=1.76 \mathrm{~T}$. The measured frequency difference between the two investigated areas is $\delta F \approx 1.3 \mathrm{GHz}$. If this frequency variation is due to a change in the anisotropy, then we obtain a variation of the anisotropy field $\delta H_{\mathrm{K}} \approx 0.145 \mathrm{~T}$ corresponding to a variation of the anisotropy constant $\delta K \approx$ $103 \mathrm{~kJ} / \mathrm{m}^{3}$.

Let us now consider the case of NB coated sample. Using the above mentioned bulk parameters, from the measured frequency variation $\delta F \approx 0.5 \mathrm{GHz}$, we obtain a variation of the anisotropy field $\delta H_{\mathrm{K}} \approx 0.048 \mathrm{~T}$ corresponding to a variation of the anisotropy constant $\delta K \approx 51 \mathrm{~kJ} / \mathrm{m}^{3}$.

Our results are consistent with the studies reporting that organic molecules affect the surface magnetic properties mainly through interfacial effects [2, 5, 6, 10-13].

Concerning the FWHM linewidth results, two possibilities arise. First possibility, the change of damping is due to interfacial spin pumping modification which is unlikely due to the weak electronic density present in molecules. Second possibility, the Co magnetic properties are modified due to a change in the interfacial electronic structure due to the coupling with the molecules.
In this sense, we propose that changes in magnetization dynamics are related to the hybridization of the $\pi$-electrons of the aromatic structures with the Co surface [5, 10, 16, 17]. Indeed, it has been demonstrated theoretically that magnetic hardening or softening can be induced in the case of ferromagnetic surface interacting with nonmagnetic organic molecules containing a single $\pi$-bond [16]. Still, by means of $a b$ initio calculations [10], it has been shown that the spin polarization of a ferromagnetic surface can be locally tailored by adsorbing organic molecules containing $\pi\left(p_{z}\right)$-electrons on it through hybridization of these out-of-plane orbitals with the spin-polarized $d$ states of the metal. Moreover, this spin polarization depends on how strongly the molecules are linked to the surface. For instance, a spin polarization reversal due to the differing hybridization between Co-phthalocyanine molecules and each cobalt electrode has been evidenced in $\mathrm{Co} / \mathrm{CoPc} / \mathrm{Co}$ magnetic tunnel junctions [13]. Interface coupling was also found to strongly influence spin injection as demonstrated in the case of $(\mathrm{La}, \mathrm{Sr}) \mathrm{MnO}_{3} / \mathrm{Alq}_{3} / \mathrm{Co}$ magnetic tunnel junctions [1], or to stabilize an antiferromagnetic ordering at room temperature at the interface between a cobalt ferromagnetic layer and a paramagnetic organic manganese phthalocyanine $(\mathrm{MnPc})$ layer [12]. Interestingly, we have observed that both change of anisotropy and increase of the damping are more important with the BTB molecule than with the NB one. Spectroscopy measurements and DFT calculations have to be performed to understand the difference in the electronic structure between those two molecules over a Co surface. The efforts must be carried forward on this effect on the magnetization dynamic aspects in order to advance towards applications in ultrahigh frequency molecular spintronic devices.

\section{CONCLUSION}

We show in this work, through BLS measurements, that different organic molecules covalently coating a Co layer have different effect on magnetization anisotropy and dynamics. Two different molecules, BTB and NB, were electrocoated on Co surface through diazonium-based electrochemistry. This electrochemical coating leads to a modification of the Co film in both cases as the same chemistry mechanism is involved. However, measured magnetization properties over the coated areas depend on the molecule nature. In NB coated area, magnons show no change in the width of the BLS peaks unlike an important modification for BTB molecules coating. These results are important for the development of molecular spintronic. Further experiments are required in order to optimize the electrochemical coating procedure and then to quantify the tuning of the properties.

\section{REFERENCES}

[1] C. Barraud, P. Seneor, R. Mattana, S. Fusil, K.

Bouzehouane, C. Deranlot, P. Graziosi, L. Hueso, I. Bergenti, V. Dediu, F. Petroff, A. Fert, Unravelling the role of the interface for spin injection into organic semiconductors, Nature Physics, 6, 615-620 (2010). 
[2] M. Kimata, D. Nozaki, Y. Niimi, H. Tajima, Y. Otani, Spin relaxation mechanism in a highly doped organic polymer film, Physical Review. B, 91, 224422 (2015).

[3] B. Dieny, V. S. Speriosu, S. S. P. Parkin, B. A. Gurney, D. R. Wilhoit, D. Mauri, Physical Review B, 43, 1297 (1991).

[4]M. Mansuripur, "The physical principles of magnetooptical recording", Cambridge University Press, (1995).

[5] A. R. Rocha, V. M. Garcia-Suarez, S. W. Bailey, C. J. Lambert, J. Ferrer, S. Sanvito, Towards Molecular Spintronics, Nature Materials 4, 335 (2005).

[6] Q. Zhang, L. Yin, W. Mi and X. Wang, Large spatial polarization at benzene/LSMO spinterface: Towards Organic spintronic devices, Journal of Physics: Condensed Matter, 120, 6156 (2016).

[7] V. Al. Dediu, L. E. Hueso, I, Bergenti, C. Taliani, Spin routes in organic semiconductors, Nature Materials, 8, 707 (2009).

[8] H. J. A. Molegraaf, J. Hoffman, C.A.F. Vaz, S. Gariglio, D. Van der Marel, C.H. Ahn, Magnetoelectric effects in complex oxides with competing ground states. Advanced Materials, 21, 3470 (2009).

[9] D. Lebeugle, A. Mougin, M. Viret, D. Colson, L. Ranno, Electric field switching of the magnetic anisotropy of a ferromagnetic layer exchange coupled to the multiferroic compound $\mathrm{BiFeO}_{3}$, Physical Review Letters, 103, 257601 (2009).

[10] N. Atodiresei, J. Brede, P. Lazic, V. Caciuc, G. Hoffmann, R. Wiesendanger, S. Blügel, Design of the local Polarization at the Organic-Ferromagnetic Interface, Physical Review Letters, 105, 066601 (2010).

[11] B. Cui, C. Song, H. Mao, H. Wu, F. Li, J. Peng. G. Wang, F. Zeng, F. Pan, Magnetoelectric coupling induced by interfacial orbital reconstruction. Advanced Materials, 27, 6651 (2015).

[12] M. Gruber, F. Ibrahim, S. Boukari, H. Isshiki, L. Joly, M. Peter, M. Studniarek, V. Da Costa, H. Jabbar, V. Davesne, U. Halisdemir, J. Chen, J. Arabski, E. Otero, F. Choueikani, K. Chen, Ph. Ohresser, W. Wulfhekel, F.Scheurer, W. Weber, M. Alouani, E. Beaurepaire, M. Bowen, Exchange bias and room-temperature magnetic order in molecular layers, Nature Materials, 14, 981-984 (2015).

[13] C. Barraud, K. Bouzehouane, C. Deranlot, S. Fusil, H. Jabbar, J. Arabski, R. Rakshit, D-J. Kim, C. Kieber, S. Boukari, M. Bowen, E. Beaurepaire, P. Seneor, R. Mattana, F. Petroff, Unidirectional Spin-Dependent MoleculeFerromagnet Hybridized States Anisotropy in Cobalt Phthalocyanine Based Magnetic Tunnel Junctions, Physical Review Letters, 114, 206603 (2015).

[14] T. Fluteau, C. Bessis, C. Barraud, M. L. Della Rocca, P. Martin, J.-C. Lacroix, P. Lafarge, Tuning the thickness of electrochemically grafted layers in large area molecular junctions, Journal of Applied Physics, 116, 114509 (2014).

[15] C. Jia, A. Migliore, N. Xin, S. Huang, J. Wang, Q. Yang, S. Wang, H. Chen, D. Wang, B. Feng, Z. Liu, G. Zhang, D. H. Qu, H. Tian, M. A. Ratner, H. Q. Xu, A. Nitzan, X. Guo, Covalently bonded single molecule junctions with stable and reversible photoswitched conductivity, Science, 352 (6292) 1443 (2016).

[16] R. Friedrich, V. Caciuc, N. S. Kiselev, N. Atodiresei, S. Blügel, Chemically functionalized magnetic exchange interactions of hybrid organic-ferromagnetic metal interfaces Physical Review B, 91, 115432 (2015).

[17] R. Friedrich, V. Caciuc, N. Atodiresei, S. Blügel, Molecular induced skyhook effect for magnetic interlayer softening, Physical Review B, 92, 195407 (2015).

[18] C. F. Hermanns, K. Tarafder, M. Bernien, A. Krüger, Y.M. Chang, P.M. Oppeneer, W. Kuch, Magnetic Coupling of Porphyrin Molecules Through Graphene, Advanced Materials, 25, 3473 (2013).

[19] P. Campiglio, R. Breitwieser, V. Repain, S. Guitteny, C. Chacon, A. Bellec, J. Lagoute, Y. Girard, S. Rousset, A. Sassella, M. Imam and S. Narasimhan, Change of cobalt magnetic anisotropy and spin polarization with alkanethiolates self-assembled monolayers, New Journal of Physics, 17, 063022 (2015)

[20] T. Still, "High Frequency Acoustics in Colloid-Based Meso- and Nanostructures by Spontaneous Brillouin Light Scattering", Springer-Verlag Berlin Heidelberg (2010).

[21] S. Demokritov, E. Tsymbal, "Light scattering from spin waves in thin films and layered systems", Journal of Physics: Condensed Matter, 36, 7145 (1994).

[22] F Zighem, Y. Roussigné, S.M. Chérif, P. Moch, Spin wave modelling in arrays of ferromagnetic thin stripes: application to Brillouin light scattering in permalloy, Journal of Physics: Condensed Matter, 19, 176220 (2007).

[23] C. Fave, Y. Leroux, G. Trippé, H. Randriamahazaka, V. Noel, J.-C. Lacroix, Tunable Electrochemical Switches Based on Ultrathin Organic Films. Journal of the American Chemical Society, 129, 1890 (2007).

[24] D. Bélanger, J. Pinson, Electrografting: a powerful method for surface modification, Chemical Society Reviews 40, 3995 (2011).

[25] J. Pinson, F. Podvorica, Attachment of organic layers to conductive or semiconductive surfaces by reduction of diazonium salts, Chemical Society Reviews, 34, 429 (2005).

[26] C. Fave, N. Vincent, J. Ghilane, G. Trippé-Allard, H. Randriamahazaka, Electrochemical Switches Based on Ultrathin Organic Films: From Diode-like Behavior to Charge Transfer Transparency, The Journal of Physical Chemistry $\mathrm{C}$ 112, 18638 (2008).

[27] M. Kostylev, Interface boundary conditions for dynamic magnetization and spin wave dynamics in a ferromagnetic layer with the interface Dzyaloshinskii-Moriya interaction, Journal of Applied Physics, 115, 233902 (2014). 